\title{
Quantum theories of consciousness
}

Pylkkänen, Paavo

Routledge

2018

Pylkkänen, P 2018, Quantum theories of consciousness . in R J Gennaro (ed.) , The Routledge Handbook of Consciousness. Routledge Handbooks in Philosophy, Routledge , Abingdon, Oxon , pp. 216-231 . https://doi.org/10.4324/9781315676982-17

http://hdl.handle.net/10138/310680

https://doi.org/10.4324/9781315676982-17

acceptedVersion

Downloaded from Helda, University of Helsinki institutional repository.

This is an electronic reprint of the original article.

This reprint may differ from the original in pagination and typographic detail.

Please cite the original version. 
Author's version, published in R. Gennaro ed. The Routledge Handbook of Consciousness. London: Routledge, 2018.

\section{Chapter 16}

\section{Quantum Theories of Consciousness}

\section{Paavo Pylkkänen}

“...quantum consciousness theory offers not just a solution to the mind-body problem, or additionally, to the nature of life and of time... And it does not just solve the Agent-Structure and Explanation-Understanding problems, or explain quantum decision theory's success in predicting otherwise anomalous behavior. What the theory offers is all of these things and more, and with them a unification of physical and social ontology that gives the human experience a home in the universe. With its elegance ... comes not just extraordinary explanatory power, but extraordinary meaning, which at least this situated observer finds utterly lacking in the classical worldview. ... I hope I have given you reason to suspend your belief that we really are just classical machines, and thus to suspend your disbelief in quantum consciousness long enough to try assuming it in your work. If you do, perhaps you will find your own home in the universe too" (Alexander Wendt, Quantum Mind and Social Science, 2015: 293).

\section{Introduction}

Much of contemporary philosophy of mind and cognitive neuroscience presupposes that the physical framework to use when explaining mind and consciousness is the framework of classical physics (and neurophysiological and/or computational processes embedded in this framework); it is typically assumed that no ideas from quantum theory or relativity theory are needed. Of course, not all theories of 
consciousness are trying to reduce conscious experience to mechanistic physical interactions at the neural level, but this tacit commitment to the classical physics of Newton and Maxwell introduces a strong mechanistic element into contemporary theorizing about consciousness, at least whenever the theories make a reference to physical processes.

One could argue that much of mainstream consciousness studies is an attempt to "domesticate" the radically non-mechanistic and experiental features of conscious experience by trying to force them to fit into the mechanistic framework (cf. Ladyman and Ross 2007: 1-2). Some researchers are happy to assume that people are just very complicated machines, or even (philosophical) zombies - machines who think they are conscious, while in fact they are just walking computers, with no such exotic features as qualia, subjectivity, experiencing and the like. Others feel that consciousness remains unexplained rather than explained by these mechanistic explanatory attempts, but even they cannot come up with a view that coherently unites conscious experience and physical processes. Thus some kind of uneasy dualism of the mental and the physical (whether acknowledged or not) often looms in those theories of consciousness that take conscious experience seriously.

However, it has been known since the early $20^{\text {th }}$ century that classical physics provides a very limited, albeit useful, description of the physical world. Classical physics fails completely in certain important domains; at high speeds and with large masses we need special and general theories of relativity (respectively), and at the atomic level, we need quantum theory. Because of experimentally detected features such as the indivisibility of the quantum of action, wave-particle duality and non- 
locality (to be briefly explained below), it can be argued that quantum theory requires a holistic rather than a mechanistic picture of reality. The mechanistic world of relatively independent objects that we find in everyday experience is then a special, limiting case that arises from a more fundamental dynamical ground in which wholeness prevails (Bohm 1980 ch. 7; Pylkkänen 2007).

It is widely agreed that conscious experience has dynamical and holistic features. Could it be that these features are in some way a reflection of the dynamic and holistic quantum physical processes associated with the brain that could underlie (and make possible) the more mechanistic neurophysiological processes that contemporary cognitive neuroscience is measuring? If so, these macroscopic processes would be a kind of shadow, or amplification of the results of quantum processes at a deeper, possibly pre-spatial level where our minds and conscious experience essentially live and unfold (cf. Penrose 1994). The macroscopic, mechanistic level is of course necessary for communication, cognition and life as we know it, including science; but perhaps the experiencing (consciousness) of that world and the initiation of our actions takes place at a more subtle, non-mechanical level of the physical world, which quantum theory has begun to discover (Bohm and Hiley 1993: 176-180).

In this chapter, after a brief historical introduction to quantum theory, we will see that the theory opens up some radically new ways of thinking about the place of mind and consciousness in nature. This need not (at least not always) deny what the other theories of consciousness are saying, but also complement them. At the very least a quantum perspective will help a "classical" consciousness theorist to become better aware of some of the hidden assumptions in his or her approach. Given that 
consciousness is widely thought to be a "hard" problem (Chalmers 1996), its solution may well require us to question and revise some of our assumptions that now seem to us completely obvious. This is what quantum theory is all about - learning, on the basis of scientific experiments, to question the "obvious" truths about the nature of the physical world and to come up with more coherent alternatives.

\section{Quantum Theory: A Brief Introduction}

Quantum theory originated at the turn of the $19^{\text {th }}$ and $20^{\text {th }}$ centuries when Planck and Einstein were studying certain experiments in which matter exchanged energy with the electromagnetic field (this section relies mostly on Bohm 1984: 70-84 and Ney 2013). Classical physics assumed that matter is composed of bodies that move continuously (determined by Newton's laws), while light consists of waves in the electromagnetic field (determined by Maxwell's equations). This implies that matter and light should exchange energy in a continuous fashion. However, to explain the photoelectric effect (in which a beam of light ejects electrons from within a metal) Einstein postulated in 1905 that light transmits energy to matter in the form of small indivisible particles or "quanta". Planck had a few years earlier postulated the existence of such quanta when explaining black-body radiation; thus the theory was to be called "quantum theory."

The above did not, however, mean that the wave nature of light that had been experimentally detected already in 1801 in Young's two-slit interference experiment was given up. On the contrary, the energy of a "particle" of light was given by the famous Planck-Einstein equation $E=h f$, where $h$ is Planck's constant and $f$ is the 
frequency of the light. Thus, the energy of a particle of light depends on the frequency of the wave aspect of the same light. Light thus has both wave and particle properties, and this somewhat paradoxical feature is called wave-particle duality.

Quantization of energy was also postulated in Bohr's 1913 model of the atom, to explain the discrete spectra emitted by a gas of, say, hydrogen. In this model a hydrogen atom consists of a proton in the nucleus, and an electron orbiting it. Bohr postulated that only certain energy levels are allowed for the electron, and when the electron jumps from a higher to a lower level, it emits a quantum of light with $E=h f$. Conversely, in order to jump from a lower to a higher level it needs to absorb a quantum of a suitable energy. A limited number of allowed energy levels implies a limited number of possible jumps, which in turn gives rise to the discrete spectral lines that had been observed.

It became possible to explain the discrete (quantized) energies of atomic orbits when de Broglie postulated in 1923 that atomic particles have a wave associated with them (Wheaton 2009). This implies that wave-particle duality applies to all manifestations of matter and energy, not just to light. In an enclosure, such as when confined within an atom, such a wave associated with an electron would vibrate in discrete frequencies (a bit like a guitar string), and if we assume that the Planck-Einstein relation $E=h f$ holds for de Broglie's waves, then discrete frequencies imply discrete energy levels, as in Bohr's model (Bohm 1984: 76).

Finally, Schrödinger discovered in 1926 an equation that determines the future motion of de Broglie's waves (which are mathematically described by a complex wave 
function $\psi)$, much in the same way as in classical physics Maxwell's equations determine the future motions of electromagnetic waves. One puzzle was how the wave function ought to be interpreted. Schrödinger was hoping to give it a physical interpretation, but did not manage to do this at the time. Max Born suggested in 1926 that the wave function describes a probability density for finding the electron at a certain region. More precisely the probability density $\rho$ at a given region is given by the square of the absolute value of the wave function, or the probability amplitude $\mid \psi$ $\left.\right|^{2}$ in that region, which is known as the Born rule $\rho=|\psi|^{2}$.

Another important development was Heisenberg's uncertainty principle. If, in a given moment, we want to measure both the position $(x)$ and the momentum $(p)$ of a particle, the uncertainty principle gives (roughly) the maximal possible accuracy $\Delta p \Delta x \geq h(\Delta p$ is uncertainty about momentum, $\Delta x$ is uncertainty about position, $h$ is Planck's constant, also known as the quantum of action, where action $h=E t$ ). This limits what we can know about a particle. But how should we interpret the uncertainty principle? Does the electron always have a well-defined position and momentum, but it is for some reason difficult for us to get knowledge about them at the same time (the epistemic interpretation)? Or does the electron not even have simultaneously a well-defined position and momentum (the ontological interpretation)? (von Wright 1989)

To observe an electron with light, we need at least one light quantum, with the energy $E=h f$. Bohr assumed that such a quantum (or more precisely the quantum of action $h=E t)$ is indivisible, and its consequences in each measurement are unpredictable and uncontrollable. Because of such nature of the quantum link in each measurement, 
Bohr said that the form of the experimental conditions and the meaning of the experimental results are a whole that is not further analyzable. This whole constitutes what Bohr called the "quantum phenomenon." Such wholeness means that the results of experiment cannot be ascribed to the properties of a particle that is assumed to exist independently of the rest of the quantum phenomenon. So Bohr interpreted the uncertainty principle in an ontological sense. We cannot define the state of being of the observed system because this state is inherently ambiguous. Depending on the experimental set-up we can apply either the concept of position or momentum. But these concepts are complementary: incompatible yet both necessary for a full description of the possible quantum phenomena. The situation is very different from that in classical physics (Bohm and Hiley 1993: 13-17; Faye 2014; Plotnitsky 2010; Pylkkänen 2015).

In 1935 Schrödinger drew attention to a curious holistic feature of quantum mechanics, which he called Verschränkung, later translated as "entanglement". This played a key role in the 1935 thought experiment by Einstein, Podolsky and Rosen (EPR). Bohr had said that because of the uncertainty principle it is meaningless to talk about an electron as if this had simultaneously a well-defined momentum and position. However, quantum mechanics implies that there are quite generally situations where two systems that interact with each other can become entangled. EPR pointed out that if two such entangled systems are separated from each other, their properties remain correlated in such a way that by measuring the position of a particle A one can obtain information about the position of particle $\mathrm{B}$, and the same for momentum - and according to them this happens "without in any way directly influencing B.” But surely, argued EPR, the particle B must have both a well-defined 
position and a well-defined momentum already prior to measurement, if an experimenter can choose which one of these she wants to measure (i.e., an experimenter can choose to measure either the position or the momentum of particle A, and in this way (without disturbing B) get information about either the position or the momentum of particle B; so surely particle B must have these properties welldefined, waiting to be revealed?). EPR concluded that quantum theory is incomplete, as it cannot account for the simultaneous existence of the position and momentum of particle B, i.e. properties which they thought that obviously exist.

Bohr's reply to EPR emphasized that we should not, like EPR did, attribute properties to particle $\mathrm{B}$, conceived in isolation from a particular quantum phenomenon involving a particular experimental set-up (see Fine 2016).

But for those physicists who think that quantum theory describes a world that exists independently of the observer, entanglement implies that experimental interventions at subsystem A influence subsystem B instantaneously, without any mediating local contact between them. Because relativity requires that signals cannot be transmitted faster than the speed of light, Einstein considered such non-locality "spooky," but experiments seem to imply a non-locality in nature (see Aspect et al. 1982; Bricmont 2016, ch. 4). We will return to the issue of non-locality below in connection with the Bohm interpretation of quantum theory.

A better understanding of some of the above ideas can be obtained by considering the famous two-slit experiment. When classical particles (e.g. bullets) pass through a wall with one or two slits in it, they build up either one or two piles on the detecting 
screen, depending on whether one or two slits are open. With waves the situation is different. If the size of the slit is roughly the same as that of the wavelength, the wave will bend or diffract after it passes through the slit. With two slits open, the diffracted waves from the two slits will meet and interfere with each other, giving rise to an interference pattern where areas where the waves add to produce a wave of large amplitude alternate with areas where the waves cancel each other out.

What happens with electrons with two slits open? The electron has typical particle properties such as mass and charge, so physicists expected that it should behave like a little bullet. However, the electrons collectively build up an interference pattern (Tonomura et al. 1989). They appear at the plate one by one at localized points, which suggests that they are particles. But it seems that each individual electron also has wave-like properties - for how else could the individual systems "co-operate" to build up an interference pattern? Note that we get an interference pattern even if we send just one electron at a time, so the pattern is not produced by the electrons interacting with each other. (For an entertaining video demonstration of the two-slit experiment, see e.g. Dr. Quantum's lecture at https://www.youtube.com/watch?v=DfPeprQ7oGc. The lecture is an excerpt from the film What The Bleep: Down The Rabbit Hole. There is some simplification and interpretation in the demo, but it gives a nice visual illustration of the experiment.)

Let us now see what the different interpretations of quantum theory say about situations like the two-slit experiment, and also consider what kind of theories of mind and consciousness some interpretations have inspired. 


\section{The Bohr Interpretation}

We have already discussed Bohr's views, so I will describe them only briefly here. Bohr said in a minimalist way that we should think of the wave function merely as a mathematical tool, as a part of an algorithm we use to calculate probabilities for the observed results of experiments. So, in the two-slit experiment we can use the Born rule to obtain probabilities for where the spots will appear in the photographic plate. As we have seen, Bohr's interpretation is very subtle and emphasizes the unanalyzable wholeness of a quantum phenomenon. Bohr did suggest that quantum theory could be relevant to understanding biological systems and even the mind (see e.g. Bohr 1934: 99), and his writings inspired others to start thinking about such issues; but as Bohr did not advance a detailed quantum theory of mind or consciousness we will not consider his view here further.

\section{Von Neumann's Interpretation: Consciousness Collapses the Wave Function}

Other physicists, such as Dirac and von Neumann, assumed that the quantum theory describes quantum reality, saying that the wave function provides the most complete possible description of the so-called "quantum state" of the electron. Bohm and Hiley (1993: 20) provide a succinct description of von Neumann's (1955) view of the quantum state and its relation to the large-scale level where we observe the results of measurement:

"This state could only be manifested in phenomena at a large scale (classical) level. Thus he was led to make a distinction between the quantum and 
classical levels. Between them, he said there was a 'cut.' This is, of course, purely abstract because von Neumann admitted, along with physicists in general, that the quantum and classical levels had to exist in what was basically one world. However, for the sake of analysis one could talk about these two different levels and treat them as being in interaction. The effect of this interaction was to produce at the classical level a certain observable experimental result. ... But reciprocally, this interaction produced an effect on the quantum level; that is the wave function changed from its original form $\psi$ to $\psi_{n}$, where $n$ is the actual result of the measurement obtained at the classical level. This change has been described as a 'collapse' of the wave function. Such a collapse would violate Schrödinger's equation, which must hold for any quantum system. However, this does not seem to have disturbed von Neumann unduly, probably because one could think that in its interaction with the classical level such a system need not satisfy the laws that apply when it is isolated."

So note that two changes take place as a result of the interaction between the quantum level and the classical level. On the one hand there will be an observable effect (e.g. a macroscopic pointer pointing to a given value) at the classical level. On the other hand, it is assumed that at the quantum level the wave function will collapse from what typically is a superposition of many possible states to a single state (a so called "eigenstate"). Note also that the terms "quantum state" and "wave function" are used interchangeably in the above quote, which is common in the discussion about the quantum theory. In this way of talking the term "wave function" is taken to refer to 
the physical quantum field that exists objectively in some sense, and not merely to a piece of mathematics.

However, there is a problem in von Neumann's approach. It is not clear what causes the collapse, because von Neumann thought that the location of the cut between the quantum level and the classical level was arbitrary. He thought that we can in principle include the observed quantum object and the measuring apparatus as part of a single combined system which has to be treated quantum mechanically (Bohm and Hiley 1993: 20). To bring about the collapse of the wave function of this combined system we then need to bring in a second measuring apparatus at the classical level to interact with the combined quantum system. But because the place of the cut is arbitrary, even this second apparatus can be included in the combined system, which requires that we introduce yet another classical apparatus, if we want to bring about a collapse, and so on. If we keep going we realize that even the brain of the observer could in principle be included in the combined quantum system. However, at the end of the experiment we experience a definite outcome rather than a complex superposition of possible states, so it seems obvious that a collapse has taken place somehow. But how could the collapse possibly happen anywhere in the physical domain, given that the cut between the quantum and classical levels is arbitrary and can be moved indefinitely? This, essentially, is the (in)famous measurement problem of quantum theory.

Given this problem, von Neumann and Wigner (1961) were led to speculate that it is only when we bring in something non-physical, namely the consciousness of the observer, that we need not apply a non-collapsed wave function $\psi$ and we get the 
definite outcome (e.g. a spot at a definite location $n$ ) we observe and can then describe the quantum system with the collapsed wave function $\psi_{n}$. This idea that it is only consciousness that can cause the collapse of the wave function and thus account for the well-defined physical reality we find in every-day experience is a historically important suggestion about the role of consciousness in quantum theory (for a critical discussion of von Neumann's and Wigner's ideas, see e.g. Bohm and Hiley 1993: 1924; see also Stapp 1993).

In recent years the von Neumann-Wigner approach has been advocated and modified especially by Henry Stapp. Alexander Wendt (2015) provides a succinct summary of Stapp's (2001) approach:

"Whereas Wigner argued that consciousness causes collapse, Stapp sees the role of the mind here as more passive, as coming to know the answer nature returns to a question. Importantly, the two roles of the mind both involve the brain/mind complex. In contrast to Cartesian dualism, therefore, Stapp's ontology is more like a psycho-physical duality or parallelism, in which every quantum event is actually a pair: a physical event in an entangled brain-world quantum system that reduces the wave function to an outcome compatible with an associated (not causal) psychical event in the mind" (Wendt 2015: 84).

The above implies that the collapse takes place without consciousness playing a causal role. It is not possible here to enter into a detailed analysis of Stapp's view, but Wendt's summary indicates that he has developed the approach in subtle ways (see also Atmanspacher 2015). 


\section{Penrose and Hameroff: quantum collapse constitutes consciousness}

Later on physicists such as Ghirardi, Rimini and Weber (1986), as well as Diosi (1989) and Penrose (1996) have developed concrete physical models about how the collapse of the quantum state happens objectively, without the consciousness of the observer having to play any role. Typically this type of theory involves introducing a mathematically described mechanism which accounts for the collapse in situations where we expect there to be just one outcome (rather than a number of possibilities typically implied by the description in terms of an uncollapsed wave function that obeys the Schrödinger equation). Thus, in the two-slit experiment we may say - in a somewhat simplified way - that the electron is a wave (described by the wave function) when it moves, but when it interacts with matter in the photographic plate, the wave collapses into a small region with a probability that obeys the Born rule and we observe a definite outcome. While this type of theory aims to show that there is no need for consciousness for there to be definite outcomes, for Penrose and Hameroff a certain kind of quantum collapse constitutes moments of conscious experience, and thus plays a key role in their quantum theory of consciousness. Let us now briefly examine this theory.

In his book The Emperor's New Mind Penrose was concerned with the physical underpinnings of human mathematical insight or understanding (Penrose 1989). Reflecting upon Gödel's theorem he was led to propose that human conscious understanding is non-computable. As he wanted to avoid the dualism of mind and matter, the question then became what sort non-computable physical process could 
underlie mathematical insight. After considering some possibilities, he suggested that the most likely candidate would be a certain kind of collapse or reduction of the quantum state. However, this would not be the usual random collapse of the quantum state (which obeys the Born rule), but rather a more subtle kind of collapse induced by gravity in some circumstances, or what Penrose later called an orchestrated objective reduction - "Orch-Or" for short. ${ }^{1}$

The question then arose concerning where in the brain such a collapse could possibly be taking place. The kind of large-scale coherent quantum states that Penrose needed in his model are fragile, and would, it seemed, be easily destroyed by the so called environmental decoherence taking place in the warm, wet and noisy environment of the human brain. There should thus be some way in which the coherent quantum states could be protected from decoherence, so that they would survive long enough and then collapse in a suitable way to properly underlie conscious understanding in the way Penrose's model had proposed. Penrose was aware that Fröhlich (1968) had suggested that there should be vibrational effects within active cells as a result of a biological quantum coherence phenomenon. These effects were supposed to arise from the existence of a large energy of metabolic energy and should not need a low temperature (Penrose 1994: 352).

Penrose then discovered that the anesthesiologist Stuart Hameroff had suggested that a computation-like action takes place within the microtubules in the cytoskeleton of neurons (Hameroff and Watt 1982; Hameroff 1987). Could such microtubules be a sufficiently protected site in the brain where the kind of large-scale quantum-coherent behavior and collapse, proposed by Penrose to underlie conscious understanding, 
might happen? Penrose and Hameroff teamed up and proposed in the mid 1990's the Orch-Or theory of consciousness, which today is the best-known quantum theory of consciousness. In a 2014 review article Hameroff and Penrose summarize their proposal:

“...consciousness depends on biologically 'orchestrated' coherent quantum processes in collections of microtubules within brain neurons, $[\ldots]$ these quantum processes correlate with, and regulate, neuronal synaptic and membrane activity, and [...] the continuous Schrödinger evolution of each such process terminates in accordance with the specific Diósi-Penrose scheme of 'objective reduction' of the quantum state. This orchestrated OR activity ('Orch Or') is taken to result in moments of conscious awareness and/or choice" (Hameroff and Penrose 2014: 39).

Note that this provides a concrete suggestion for a mechanism for how the "quantum mind" could influence (and be influenced by) the large-scale, classical neural processes that mainstream cognitive neuroscience is focusing upon.

There have been many criticisms of the Penrose-Hameroff proposal, often in prestigious scientific journals, for example by Grush and Churchland (1995), Tegmark (2000a and 2000b), Litt et al. (2006), Koch and Hepp (2006), Reimers et al. (2009) and McKemmish et al. (2009). However, Hameroff and Penrose have provided detailed responses to the criticisms, and the theory still remains a live option, albeit an exotic one (for a summary of and references to their replies see their 2014: 66-68; for discussion see Wendt 2015: 102-108). 


\section{Everett's Many Worlds Interpretation}

Yet other physicists have tried to account for the experimental quantum phenomena without postulating a collapse. One radical possibility is to follow Everett (1954) and assume that in each situation where the wave function implies a number of possible outcomes, but we perceive only one outcome (e.g. an electron at point $n$ ), there is no collapse of the quantum state, but instead the world at a macroscopic level branches into copies so that there is a branch corresponding to each possible outcome. So with two possible outcomes $(\mathrm{x}=1$ or $\mathrm{x}=2)$ the world branches into two copies that differ in that in one of them the macroscopic pointer indicates, say, that the electron is at point $\mathrm{x}=1$ (which the observer in that branch sees) and in the other one it is at point $\mathrm{x}$ $=2$ (which the observer in that branch sees), and so on. In the two-slit experiment there are a large number of possible places where the electron can be detected, and correspondingly the world branches into a large number of copies each time an electron is detected (Lewis 2016: 6). While this "many worlds" interpretation may sound very implausible, some physicists find it attractive because they think it best reflects the experimentally verified Schrödinger equation and has also other virtues (Saunders et al. 2010; Wallace 2012). Some researchers have even proposed in the context of the Everett theory that each conscious brain is associated with many minds, where some of the minds follow each branch! (Albert and Loewer 1988; Lockwood 1989; 1996; for discussion see Lewis 2015: 132-133).

\section{The Bohm Interpretation: The Wave Function Describes Active Information}


Yet another interpretation which avoids the need to postulate a collapse is due to de Broglie (1927) and Bohm (1952 a, b). This assumes that the electron is a particle always accompanied by a new type of quantum field, described by the wave function. We will focus on Bohm and Hiley's $(1987,1993)$ version of the de Broglie-Bohm interpretation and will call it hereafter "the Bohm theory" (for de Broglie's views, see Bacciagaluppi and Valentini 2009).

In the Bohm theory the field gives rise to a quantum potential which influences the movement of the particle, besides classical potentials, and in this way gives rise to quantum effects. Let us see how the theory deals with the two-slit experiment. In Figure 16.1 the particles are coming towards us from the two slits. When a particle passes a slit it will encounter the quantum potential which arises from the quantum field that has passed both slits and interfered with itself. One can think of a potential as a bit analogous to a mountain, so that the quantum potential will, for example, keep the electrons away from areas where it has a high value.

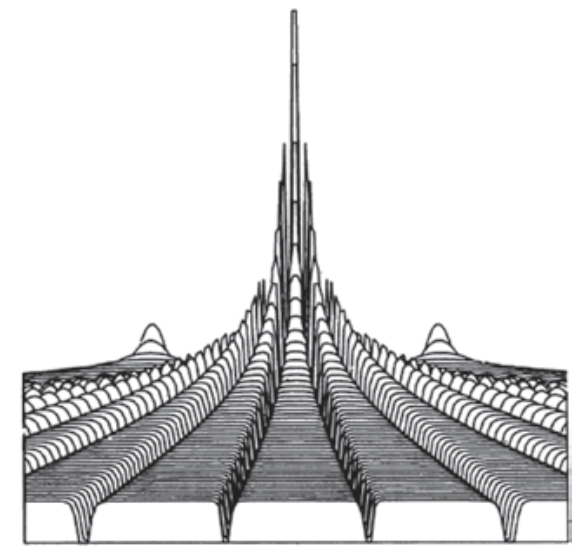

Figure 16.1 Quantum Potential for Two Gaussian Slits (from Philippidis, Dewdney and Hiley 1979). Reprinted with kind permission of Società Italiana di Fisica, copyright (1979) by the Italian Physical Society (https://link.springer.com/article/10.1007\%2FBF02743566) 
The particles (electrons) have their source in a hot filament, which means that there is a random statistical variation in their initial positions. This means that each particle typically enters the slit system in a different place. The Bohm theory assumes that this variation in the initial positions is typically consistent with the Born rule, so that the theory gives the same statistical predictions as the usual quantum theory. Figure 16.2 shows some possible trajectories than an electron can take after it goes through one of the slits. Which trajectory it takes depends, of course, on which place it happens to enter the slit system. The theory provides an explanation of the two-slit experiment without postulating a collapse of the wave function.

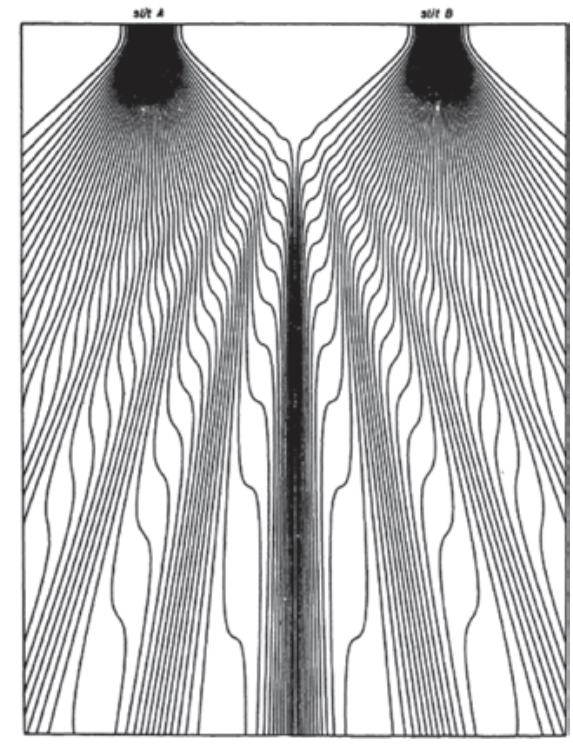

Figure 16.2 Trajectories for Two Gaussian Slits (from Philippidis, Dewdney and Hiley 1979) with kind permission of Società Italiana di Fisica, copyright (1979) by the Italian Physical Society (https://link.springer.com/article/10.1007\%2FBF02743566)

Note that the trajectories in the Bohm theory should be seen as a hypothesis about what may be going on in, say, the two-slit experiment. Because of the uncertainty principle we are not able to observe the movement of individual quantum particles. However, there is currently an attempt to experimentally determine the average trajectories of atoms by making use of the measurements of so-called weak values 
(Flack and Hiley 2014). Over the years there have been many criticisms of the de Broglie-Bohm interpretation, but its proponents have been able to provide answers (see Goldstein 2013; Bricmont 2016).

When Bohm re-examined his 1952 theory with Basil Hiley in the early 1980s, he considered the mathematical form of the quantum potential. With classical waves the effect of the wave upon a particle is proportional to the amplitude or size of the wave. However, in Bohm's theory the effect of the quantum wave depends only upon the form of the quantum wave, not on its amplitude (mathematically, the quantum potential depends upon the second spatial derivative of the amplitude). Bohm realized that this feature might be revealing us something important about the nature of quantum reality. For instead of saying that the quantum wave pushes and pulls the particle mechanically, the mathematics suggests that the form of the quantum field is literally in-forming the energy of the particle. This is somewhat analogous to the way a radar wave guides a ship on autopilot. The radar wave is not pushing and pulling the ship, but rather the form of the radar wave (which reflects the form of the environment) informs the greater energy of the ship. Analogously, Bohm thought that the quantum field carries information about the form of the environment (e.g. the presence of slits) and this information directs the particle to move in a particular way.

Another puzzling feature in quantum mechanics (and also in Bohm's theory) is that the wave function for a many-body system necessarily lives in a $3 \mathrm{~N}$-dimensional configuration space (where $\mathrm{N}$ is the number of particles in a system). So for a twoparticle entangled system the wave lives in a 6-dimensional space, and so on. But how could one possibly give a physical interpretation to such a multidimensional field? 
This was not a problem for Niels Bohr, because he thought we should not give an ontological interpretation to the wave function in the first place. But approaches that assume that the wave function describes reality have to deal with this issue of multidimensionality (for a discussion, see Ney and Albert 2013).

The idea of active information also helps to make sense of this multidimensionality, for it is common to think that information can be organized multi-dimensionally. If the essential nature of the quantum field is information, then it is perhaps not such a mystery that it is organized in a multi-dimensional way. This does not mean that Bohm's suggestion is not exotic - for one thing the Bohmian multidimensional information mediates non-local correlations through the quantum potential. But as was mentioned above, experiments indicate that there exists some kind of quantum non-locality in nature. This seems to create a tension with relativity according to which it is not possible to signals faster than the speed of light. However, Bohm and Hiley point out that it is not possible to send signals non-locally by modulating the wave function (1993: 282-284). Also, recent research by Walleczek and Grössing (2016) shows how a certain kind of non-local information transfer can be compatible with the theory of relativity.

Bohm and Hiley's proposal about active information has not always been received enthusiastically in the physics community (see e.g. Riggs 2008). However, some leading thinkers take it seriously (e.g. Holland 1993; Smith 2003; Khrennikov 2004). Note also that there exists a more minimalist version of the Bohm theory known as "Bohmian mechanics" which does not give the quantum potential a great significance (and thus usually ignores the notion of active information). For this approach which 
has some support among philosophers of physics, see Goldstein 2013; Bricmont 2016; Bell 1987; for a discussion, see Holland 2011).

Bohm had been interested in the possible relevance of quantum theory to understanding the nature of mind and consciousness already in his 1951 textbook Quantum theory, pointing to some striking analogies between quantum processes and thought (Bohm 1951: 168-172; Pylkkänen 2014). In the 1960s he developed a more general framework for physics, which he called the implicate order. The notion of the implicate order tries to capture the flowing, undivided wholeness of quantum and relativistic phenomena, and Bohm also applied it to describe the holistic and dynamic features of conscious experience, such as time consciousness (Bohm 1980, 1987; Pylkkänen 2007). In a similar vein, he thought that the notion of active information is relevant to understanding the relationship between mind and matter. He proposed that the active information carried by the quantum field could be seen as a primitive mindlike quality of, say, an electron. This sounds like a panpsychist move, but Bohm thought it was obvious that an electron does not have consciousness, and was thus not embracing panpsychism in the traditional sense which attributes experience to the ultimate constituents of the world (Bohm 1989; 1990; Pylkkänen, forthcoming; cf. Strawson 2006a, b).

How might the above be relevant to the mind-matter problem? Bohm and Hiley suggested that it is natural to extend the quantum ontology (1993: 380). So just as there is a quantum field which informs the motion of the particle, there could be a super-quantum field which informs the movement of the first-order quantum field, and so on. Bohm speculated that the information in our mental states could be a part 
of the information contained in this hierarchy of fields of quantum information. This way the information in our mental states could influence neural processes by reaching the quantum particles and/or fields in a suitable part of the brain (e.g. in synapses or microtubules or other suitable sites, to be revealed by future quantum brain theory). In effect, Bohm was proposing a solution to the problem of mental causation. ${ }^{2}$

\section{Explaining Qualia in a Quantum Framework}

We have above given a brief introduction to some aspects of quantum theory, as well as to some quantum theories of mind and consciousness. However, the above only gives a small glimpse of the great variety and diversity of such theories. In this section we will approach the question differently, by taking up an essential feature of consciousness, namely qualia, and considering how some of the quantum approaches might help to explain them.

Presumably the most discussed and debated feature of conscious experience is its qualitative character - the blueness of the sky, the taste of chocolate, and similar sensory qualia. Do quantum theories of consciousness have anything to say about qualia? In further developments of their theory, Hameroff and Penrose have introduced an explicitly panpsychist element to it. For they (2014: 49) note that the Diósi-Penrose proposal suggests that "each OR [objective reduction] event, which is a purely physical process, is itself a primitive kind of 'observation,' a moment of 'proto-conscious experience'." They (2014: 72) further elaborate this idea: “...in the Orch OR scheme, these [non-orchestrated OR] events are taken to have a rudimentary subjective experience, which is undifferentiated and lacking in cognition, perhaps 
providing the constitutive ingredients of what philosophers call qualia." The idea is that the unorchestrated and ubiquitous objective reductions involve proto-qualia, but when such reductions are orchestrated (e.g. in the human brain), then qualia in a full sense emerge. Of course, this idea may sound very speculative and even ad hoc; but given that very little can be said about the origin of qualia in the mechanistic classical physical framework of mainstream neuroscience, perhaps one should keep an open mind here.

Also, we saw above how Bohm and Hiley proposed that the wave function describes a field of active information, which can be seen as a primitive mind-like quality of the particle. The idea of quantum theoretical active information is perhaps most naturally seen as proposing that electrons have "proto-cognition" (because of the information aspect) and "proto-will" (because the information is fundamentally active) (cf. Wendt 2015: 139). But in search of a panpsychist solution to the hard problem of consciousness one could also, somewhat similar to Chalmers's (1996) double-aspect theory of information, postulate that Bohmian quantum theoretical active information has proto-phenomenal and proto-qualitative aspects. Such proto-qualia could be the content of such active information, a kind of "proto-meaning" that active information has for the electron (cf. Pylkkänen 2007: 244-246). Again, this is very speculative, but the basic idea is that the quantum ontology with its subtle, non-classical properties provides the ground from which qualia in a full sense might emerge, in a suitably organized biological or artificial system.

\section{Quantum Biology, Quantum Cognition and Quantum Computation}


The attempt to explain mind and consciousness in terms of the quantum theory involves heavy speculation - can we really cross the explanatory gap with a quantum leap? While we may not be able to answer that question in the near future, it is worth noting that in recent years we have seen significant advances in other areas where the ideas and formalisms of quantum theory have been applied to new domains. In biology, it has been shown how quantum effects (e.g. quantum coherent energy transfer and entanglement) are likely to play a role photosynthesis and avian magnetoreception (Ball 2011; Lambert et al. 2013). Lambert et al. (2013: 16) conclude their review article of quantum biology in Nature Physics as follows:

"The fact that there is even the possibility of a functional role for quantum mechanics in all of these systems suggests that the field of quantum biology is entering a new stage. There may be many more examples of functional quantum behavior waiting to be discovered."

These advances in quantum biology, while not giving direct support to quantum brain theory, perhaps make a biologically grounded quantum theory of consciousness seem less inconceivable.

Another area where there has been interesting cutting-edge research is quantum cognition (sometimes also called "quantum interaction"). In recent years a number of researchers have proposed that certain principles and mathematical tools of quantum theory (such as quantum probability, entanglement, non-commutativity, non-Boolean logic and complementarity) provide a good way of modeling many significant cognitive phenomena (such as decision processes, ambiguous perception, meaning in 
natural languages, probability judgments, order effects and memory; for an introduction, see Wang et al. 2013; Pothos and Busemeyer 2013; Busemeyer and Bruza 2012). While quantum cognition researchers are typically agnostic regarding whether there are any significant quantum effects in the neural processes underlying cognition, it can be argued that the success of quantum cognition also provides support for the stronger quantum mind and consciousness programs (Wendt 2015: 154-155).

Finally, there has been significant research in areas such as quantum information, computation and cryptography, providing yet another example where it has been valuable to apply quantum theory to new domains (Bouwmeester et al. 2000)

There are a number of important quantum approaches to mind and consciousness that we have not covered in this short review. There is the quantum field theoretical program that involves a quantum view of memory, going back to Umezawa and Ricciardi (Ricciardi and Umezawa 1967; Jibu and Yasue 1995; Vitiello 2001; Globus 2003; for a succinct account see Atmanspacher 2015). There is also Beck and Eccles's (1992) proposal that synaptic exocytosis can be controlled by a quantum mechanism (see Atmanspacher 2015; Hiley and Pylkkänen 2005). Eccles saw this proposal as opening up a way for the (non-physical) self to control its brain without violating the energy conservation laws. In a recent development, the physicist Matthew Fisher has given support to a strong version of quantum cognition by proposing that quantum processing with nuclear spin might be operative in the brain (Fischer 2015). There are also interesting approaches that see quantum theory as grounding a double-aspect view of mind and matter and which have been inspired by 
the ideas of Jung and Pauli (Atmanspacher 2014; 2015).

Many tend to dismiss quantum theories of consciousness as too speculative and implausible. Others, however, hold that it is only through such radical thinking, guided by our best scientific theories, that we will ever make progress with the harder problems of mind and consciousness.

\section{Related Topics}

Materialism

Dualism

Idealism, Panpsychism, and Emergentism

\section{Further reading}

Atmanspacher, H. (2015) "Quantum Approaches to Consciousness," The Stanford Encyclopedia of Philosophy (Summer 2015 Edition), E. N. Zalta (ed.), URL = $<$ http://plato.stanford.edu/archives/sum2015/entries/qt-consciousness/>. (Essential reading for anyone interested in quantum theories of consciousness; see also the many other articles on quantum theory in The Stanford Encyclopedia.)

Bohm, D. and Hiley, B.J. (1993) The Undivided Universe: An Ontological Interpretation of Quantum Theory, London: Routledge. (An attempt to make quantum theory intelligible which includes accurate descriptions and critical reflections of the views of Bohr, von Neumann, Everett, Ghirardi, Rimini \& Weber, Stapp and GellMann \& Hartle). 
Polkinghorne, J. (2002) Quantum theory: A Very Short Introduction, Oxford: Oxford University Press. (A remarkably lucid introduction to quantum theory for the uninitiated.)

Wendt, A. (2015) Quantum Mind and Social Science: Unifying Physical and Social Ontology, Cambridge: Cambridge University Press. (An insightful and comprehensive review of the revolutionary quantum mind proposals by a leading social scientist.)

\section{Bibliography}

Aspect, A., Grangier, P. and Roger, G. (1982) 'Experimental test of Bell's inequalities using time-varying analyzers," Physical Review Letters 49: 1804-1807.

Atmanspacher, H., 2014, "20th century variants of dual-aspect thinking (with commentaries and replies)," Mind and Matter 12: 245-288.

----- (2015) “Quantum Approaches to Consciousness,” The Stanford Encyclopedia of Philosophy (Summer 2015 Edition), E. N. Zalta (ed.), URL = $<$ http://plato.stanford.edu/archives/sum2015/entries/qt-consciousness/>.

Bacciagaluppi, G. and Valentini, A. (2009) Quantum Theory at a Crossroads: Reconsidering the 1927 Solvay Conference, Cambridge: Cambridge University Press.

Ball, P. (2011) “The Dawn of Quantum Biology,” Nature 474: 272-274. http://www.nature.com/news/2011/110615/full/474272a.html). 
Beck, F. and Eccles, J. (1992) "Quantum aspects of brain activity and the role of consciousness," Proceedings of the National Academy of Sciences 89 (23): 11357 11361.

Bell, J. (1987) Speakable and Unspeakable in Quantum Mechanics, Cambridge: Cambridge University Press.

Bohm, D. (1951) Quantum Theory, Englewood Cliffs: Prentice Hall. Dover edition 1989.

---- (1952 a and b) “A Suggested Interpretation of the Quantum Theory in Terms of ‘Hidden Variables' I and II,’ Physical Review 85 (2): 166-179 and 180-193.

----- (1980) Wholeness and the Implicate Order, London: Routledge.

----- (1984) Causality and Chance in Modern Physics, London: Routledge. New edition with new preface. First edition published in 1957.

---- (1987) "Hidden Variables and the Implicate Order," in B.J. Hiley and F. D. Peat (eds.) Quantum Implications: Essays in Honour of David Bohm, London: Routledge.

---- (1989) “Meaning and Information,” in P. Pylkkänen (ed.) The Search for Meaning, Wellingborough: Crucible. 
---- (1990) “A new theory of the relationship of mind and matter," Philosophical Psychology 3: 271-286.

Bohm, D. and Hiley, B. J. (1987) “An Ontological Basis for Quantum Theory: I. Nonrelativistic Particle Systems," Physics Reports 144: 323-348.

---- (1993) The Undivided Universe. An Ontological Interpretation of Quantum Theory, London: Routledge.

Bohr, N. (1934) Atomic Theory and the Description of Nature, Cambridge: Cambridge University Press (new edition 2011).

Bouwmeester, D., Ekert, A., and Zeilinger, A. K. (eds.) (2000) The Physics of Quantum Information: Quantum Cryptography, Quantum Teleportation, Quantum Computation, Heidelberg and Berlin: Springer

Bricmont, J. (2016) Making Sense of Quantum Mechanics, Heidelberg: Springer.

Busemeyer, J. and Bruza, P. (2012) Quantum Models of Cognition and Decision, Cambridge: Cambridge University Press.

Chalmers, D. (1996) The Conscious Mind: In Search of a Fundamental Theory, Oxford: Oxford University Press. 
Chrisley, R. C. (1997): “Learning in Non-superpositional Quantum Neurocomputers,” in Pylkkänen et al. 1997.

Diósi, L. (1989) "Models for universal reduction of macroscopic quantum fluctuations," Physical Review A 40: 1165-1174.

Everett, H. III (1957) “'Relative state" formulation of quantum mechanics'," reprinted in J. Wheeler and W. Zurek (eds.) (1983) Quantum theory and measurement, Princeton: Princeton University Press.

Faye, J. (2014) "Copenhagen Interpretation of Quantum Mechanics", The Stanford Encyclopedia of Philosophy (Fall 2014 Edition), E. N. Zalta (ed.), URL = $<$ https://plato.stanford.edu/archives/fall2014/entries/qm-copenhagen/>.

Fine, A. (2016) "The Einstein-Podolsky-Rosen Argument in Quantum Theory," The Stanford Encyclopedia of Philosophy (Fall 2016 Edition), E. N. Zalta (ed.), URL = $<$ https://plato.stanford.edu/archives/fall2016/entries/qt-epr/>.

Fischer, M.P.A. (2015) "Quantum cognition: The possibility of processing with nuclear spins in the brain," Annals of Physics 362, pp. 593-602.

Flack, R. and Hiley, B. J. (2014) "Weak Measurement and its Experimental Realisation," Journal of Physics: Conference Series 50. arXiv:1408.5685 
Fröhlich, H. (1968) "Long range coherence and energy storage in biological systems," International Journal of Quantum Chemistry II: 641-649.

Ghirardi, G. C., Rimini, A. and Weber, T. (1986) "Unified dynamics for microscopic and macroscopic systems," Physical Review D 34: 470.

Globus, G. (2003) Quantum Closures and Disclosures, Amsterdam: John Benjamins.

Goldstein, S. (2013) "Bohmian Mechanics," The Stanford Encyclopedia of Philosophy, E. N. Zalta (ed.), URL = http://plato.stanford.edu/archives/spr2013/entries/qm-bohm/

Grush, R. and Churchland, P.S. (1995) "Gaps in Penrose's Toilings," Journal of Consciousness Studies, 2 (1): 10-29.

Hameroff, S. (1987) Ultimate computing: Biomolecular consciousness and nanotechnology, Amsterdam: North-Holland.

Hameroff, S., and Penrose, R. (2014) "Consciousness in the universe: A review of the 'Orch OR theory'," Physics of Life Reviews 11: 39-78.

Hameroff, S. and Watt, R. (1982) “Information processing in microtubules," Journal of Theoretical Biology 98: 549-61. 
Hiley, B.J. and Pylkkänen, P. (1997) “Active information and cognitive science: A reply to Kieseppä,” in Pylkkänen et al. 1997.

----- (2005) “Can mind affect matter via active information?," Mind and Matter 3 (2):

7-26. URL = http://www.mindmatter.de/resources/pdf/hileywww.pdf

Holland, P. (1993) The Quantum Theory of Motion: An Account of the de BroglieBohm Causal Interpretation of Quantum Mechanics, Cambridge: Cambridge University Press.

----- (2011) “A Quantum of History,” Contemporary Physics 52: 355.

Jibu, M. and Yasue, K. (1995) Quantum Brain Dynamics and Consciousness, Amsterdam: John Benjamins.

Kieseppä, I. A. (1997a) “Is David Bohm’s Notion of Active Information Useful in Cognitive Science?," in Pylkkänen et al. 1997.

----- (1997b) "On the Difference between Quantum and Classical Potentials - A Reply to Hiley and Pylkkänen,” in Pylkkänen et al. 1997.

Khrennikov, A. (2004) Information Dynamics in Cognitive, Psychological and Anomalous Phenomena, Series Fundamental Theories of Physics 138, Dordrecht: Kluwer. 
Koch, C. and Hepp, K. (2006) "Quantum mechanics in the brain,” Nature 440: 661.

Ladyman, J. and Ross, D. (with Spurrett, D. and Collier, J.) (2007) Every Thing Must Go: Metaphysics Naturalized, Oxford: Oxford University Press.

Lambert, N. Chen Y.-N., Cheng, Y.-C., Li, C.-M, Chen, G.-Y. and Nori, F. (2013)

"Quantum Biology," Nature Physics 9: 10-18.

Lewis, P. (2016) Quantum Ontology: A Guide to the Metaphysics of Quantum Mechanics, Oxford: Oxford University Press.

Litt, A., Eliasmith, D., Kroon, F., Weinstein, S., and Thagard, P. (2006) "Is the Brain a Quantum Computer?,” Cognitive Science 30: 593-603.

Lockwood, M. (1989) Mind, Brain and the Quantum, Oxford: Blackwell.

---- (1996) “Many Minds Interpretations of Quantum Mechanics,” British Journal for the Philosophy of Science 47: 159-188.

McKemmish, L., Reimers, J., McKenzie, R., Mark, A., and Hush, N. (2009)

"Penrose-Hameroff Orchestrated Objective-Reduction Proposal for Human

Consciousness is not Biologically Feasible," Physical Review E 80 (2): 021912-1 to 021912-6.

Ney, A. (2013) “Introduction,” in A. Ney and D. Albert (eds.) (2013). 
Ney, A. and Albert, D. (eds.) (2013) The Wave Function: Essays on the Metaphysics of Quantum Mechanics, Oxford: Oxford University Press.

Penrose, R. (1989) The Emperor's New Mind, Oxford: Oxford University Press.

---- (1994) The Shadows of the Mind, Oxford: Oxford University Press.

----- (1996) "Wavefunction collapse as a real gravitational effect," General Relativity and Gravitation 28: 581-600.

Philippidis, C., Dewdney, C. and Hiley, B. J. (1979) "Quantum interference and the quantum potential," Il Nuovo Cimento 52: 15-28.

Plotnitsky, A. (2010) Epistemology and Probability. Bohr, Heisenberg, Schrödinger and the Nature of Quantum-Theoretical Thinking, Heidelberg and New York: Springer.

Pothos, E. M. and Busemeyer, J. R. (2013) “Can quantum probability provide a new direction for cognitive modeling?," Behavioral and Brain Sciences 36: 255-327.

Pylkkänen, P. (1995) “Mental Causation and Quantum Ontology,” Acta Philosophica Fennica 58: 335-348.

---- (2007) Mind, Matter and the Implicate Order, Berlin and New York: Springer 
Frontiers Collection.

---- (2014) "Can quantum analogies help us to understand the process of thought?," Mind and Matter 12: 61-91.

http://www.mindmatter.de/resources/pdf/pylkkaenen_www.pdf

---- (2015) “The Quantum Epoché,” Progress in Biophysics and Molecular Biology 119: $332-340$.

---- (2017) "Is there room in quantum ontology for a genuine causal role of consciousness?," in A. Khrennikov and E. Haven (eds.) The Palgrave Handbook of Quantum Models in Social Science, London: Palgrave Macmillan UK.

----- (forthcoming) “A Quantum Cure for Panphobia,” to appear in W. Seager (ed.) Routledge Companion to Panpsychism, London: Routledge.

Pylkkänen, P., Pylkkö, P. and Hautamäki, A. (eds.) (1997) Brain, Mind and Physics, Amsterdam: IOS Press.

Pylkkö, P. (1998) The Aconceptual Mind: Heideggerian Themes in Holistic Naturalism, Amsterdam: John Benjamins.

Reimers, J., McKemmish, L., McKenzie, R., Mark, A., and Hush, N. (2009) "Weak, Strong, and Coherent Regimes of Frohlich Condensation and their Applications to 
Terahertz Medicine and Quantum Consciousness," Proceedings of the National Academy of Sciences 106: 4219-4224.

Ricciardi, L. and Umezawa. H. (1967) "Brain and physics of many-body problems," Kybernetik 4 (2): 44-48.

Riggs, P. (2008) "Reflections on the de Broglie - Bohm quantum potential," Erkenntnis 68: 21-39.

Saunders, S., Barrett, J., Kent, A. and Wallace, D. (eds.) (2010) Many Worlds? Everett, Quantum Theory, \& Reality, Oxford: Oxford University Press.

Smith, Q. (2003) "Why cognitive scientists cannot ignore quantum mechanics?," in Q. Smith and A. Jokic (eds.) Consciousness: New Philosophical Perspectives, Oxford: Oxford University Press.

Stapp, H. (1993) Mind, Matter and Quantum Mechanics, Berlin: Springer Verlag.

---- (2001) “Quantum theory and the role of mind in nature," Foundations of Physics 31: 1465-1499.

Strawson, Galen (2006a) "Realistic monism - why physicalism entails panpsychism," Journal of Consciousness Studies 13 (10-11): 3-31. 
---- (2006b) "Panpsychism? Reply to commentators with a celebration of Descartes," Journal of Consciousness Studies 13 (10-11): 184-280.

Tegmark, M. (2000a) "lmportance of Quantum Decoherence in Brain Processes," Physical Review E 61 (4): 4194-4206.

----- (2000b) "Why the Brain Is Probably Not a Quantum Computer," Information Sciences 128: 155-179.

Tonomura, A., Endo, J,. Matsuda, T., Kawasaki, T. and Ezawa, H. (1989)

"Demonstration of single-electron buildup of an interference pattern," American Journal of Physics 57: 117-120.

Vitiello, G. (2001) My Double Unveiled: The Dissipative Quantum Model of the Brain, Amsterdam: John Benjamins.

von Neumann, J. (1955) Mathematical Foundations of Quantum Mechanics, Princeton: Princeton University Press. (First edition in German, Mathematische Grundlagen der Quantenmechanik, 1932.)

von Wright, G.H. (1989) "Images of science and forms of rationality," in S. J. Doorman (ed.) Images of Science: Scientific Practise and the Public, Aldershot: Gower. 
Wallace, D. (2012) The Emergent Multiverse: Quantum Theory according to the Everett Interpretation, Oxford: Oxford University Press.

Walleczek, J. and Grössing, G. (2016) “Nonlocal Quantum Information Transfer Without Superluminal Signalling and Communication," Foundations of Physics 46: $1208-1228$.

Wang, Z., Busemeyer, J. R., Atmanspacher, H., and Pothos, E. M. (2013) “The potential of using quantum theory to build models of cognition," Topics in Cognitive Science 5: 672-688.

Wheaton, B.R. (2009) "Matter Waves," in D. Greenberger, K. Hentschel and F. Weinert (eds.) Compendium of Quantum Physics: Concepts, Experiments, History and Philosophy, Berlin: Springer.

Wigner, E. (1961) "Remarks on the mind-body problem," in I.J. Good (ed.) The Scientist Speculates, London: Heinemann.

\section{END NOTES}

\footnotetext{
${ }^{1}$ See his 1994 book Shadows of the Mind for a detailed exposition of these ideas; for criticisms by a number of commentators as well as Penrose's reply, see the internet journal Psyche at http://journalpsyche.org/archive/volume-2-1995-1996/; see also Pylkkö (1998, ch. 4).
} 
2 Bohm (1990); Pylkkänen (1995; 2007; 2017); Hiley and Pylkkänen (2005); for criticisms see Kieseppä (1997 a, b) and Chrisley (1997); for a reply see Hiley and Pylkkänen (1997). 